\title{
Preventing Neonatal Legionellosis-The Environmental Surveillance Approach
}

\author{
Ioanna G Alexandropoulou ${ }^{1,2^{\star}}$, Maria Panopoulou ${ }^{2}$, Theodoros A Parasidis ${ }^{1}$, Theocharis Konstantinidis ${ }^{1}$ and Theodoros $\mathrm{C}$ Constantinidis ${ }^{1}$ \\ ${ }^{1}$ Laboratory of Hygiene and Environmental Protection, Medical School, Democritus University of Thrace, Campus (Dragana) Building , 68100 Alexandroupolis, Greece \\ ${ }^{2}$ Microbiology Laboratory, Medical School, Democritus University of Thrace, Campus (Dragana), 68100 Alexandroupolis, Greece
}

*Corresponding author: loanna G Alexandropoulou, Microbiology Laboratory, Medical School, Democritus University of Thrace, Campus (Dragana), 68100 Alexandroupolis, Greece, Tel: +30-2541-07902; E-mail: ialexand@med.duth.gr

Received date: July 09, 2015; Accepted date: July 11, 2015; Published date: July 18, 2015

Copyright: (c) 2015 Alexandropoulou IG, et al. This is an open-access article distributed under the terms of the Creative Commons Attribution License, which permits unrestricted use, distribution, and reproduction in any medium, provided the original author and source are credited.

\section{Neonatal and Pediatric Legionellosis}

Legionellosis is thought to be a very uncommon cause of pneumonia in children and even more in neonates [1]. Despite this, they have been documented cases of legionnaire's disease in pediatric patients. The majority of them were hospital-acquired and the source of infection in most cases was hospital's tap water [1,2]. In children legionellosis, the most common symptoms are fever and cough and the most common findings are tachypnoea, hypoxia and abnormal lung examination [1]. Especially neonates, due to their underdeveloped immune defenses, as well as the intensive medical treatments they may receive, represent another population that could be at particularly high risk for Legionella infection [1].

\section{The Environmental Approach-The Strategy in Greece}

Routine monitoring of hospitals tap water and especially neonatal, obstetric and pediatric units remains a controversial matter. CDC recommendations are focused on environmental monitoring, only after recognized cases of Legionnaires disease, or in transplant units [3]. In Greece there is no specific legislation for Legionella spp. But the Hellenic Ministry of Health has instituted guidelines for the prevention and control of Legionnaires' disease, based on European Working Group for Legionella Infections (EWGLI) recommendations [4]. Based on these hospitals and health care facilities, proceed in routine water samplings at least twice a year as it was described before [5-8].

\section{Review of the Literature}

A review of the medical literature by Greenberg et al. identified 76 cases of Legionella infection in children, $78 \%$ of whom had an underlying condition such as malignancy [1]. Shachor-Meyouhas et al., report that $7 / 13(54 \%)$ of neonatal who contracted legionellosis survived [9].

Only a few sporadic and one outbreak of hospital-acquired neonatal legionellosis have been reported in literature. The majority of them were linked to contaminated water. Levy and Rubin, had previous reviewed 9 cases of hospital-acquired neonatal legionellosis [2]. Six of the infants had a fatal outcome (55\% mortality). An environmental link was found at 5 cases, in 4 cases with the hospital water system and in 1 case with a humidifier and an incubator.

Greenberg et al., had review another 3 cases of hospital-acquired neonatal legionellosis with an environmental link to the hospital [1]. Another 2 cases of neonatal Legionella infection are associated with contaminated hospital water in Taiwan. In this report, Legionella isolates from neonates and from water dispensers were found indistinguishable [10]. A large outbreak has been reported in a neonatal unit of a private hospital in Cyprus, linked to hospital tap water. Eleven cases were reported and three deaths [11]. ShachorMeyouhas et al describe an 11-day-old neonate who contracted Legionnaires' disease, as it was rinsed in a hospitals' sink [9].

\section{The Antibiotic Approach-In vivo and In vitro}

Macrolides, rifampicin and flouroquinolones are the treatment of choice for legionellosis. The selection of these categories is mainly based on clinical experience [12]. The antibiotic erythromycin is the historically drug of choice for the treatment of legionellosis [13]. However, the latest years the use of fluoroquinolones is increasing because they seem to be superior in inhibiting intracellular growth of Legionella spp. both in vitro and in animal models [14,15]. It was found that strain resistance in fluoroquinolones with in vitro selection can be acquired with an 8-fold increase in MIC after three passages [16]. Susceptibility testing, as a routine procedure for Legionella strains clinical or environmental, is not commonly used. As there is no specific regulation on susceptibility testing and Legionella spp. has particular nutritional requirements, various methods have been used to determine MIC values: E-test, broth dilution, agar dilution, in vivo animals' models and in vitro cell cultures [14-18].

Based on findings, most clinical Legionella isolates are not resistant to these antibiotics, despite the fact that many studies showed in patients' failure of treatment $[17,19,20]$. Despite this, as legionellosis is acquired from water samples, susceptibility testing of environmental isolates could recognize if an antibiotic-resistant strain has colonized the water distribution system and thus increase the risk of infection [21-23]. The presence of environmental Legionella pneumophila strains that are less susceptible in antibiotics could probably increase the risk of a failed antibiotic treatment in patients with legionellosis, especially those who are in high risk groups such as neonates and infants [24].

\section{Conclusion}

Legionnaires' disease is thought to be rare in children, a fact that leads to a delay in diagnosis and to the appropriate antimicrobial therapy. As it happens in most cases of legionellosis in adults, in neonates the incidence can also be underestimated. The main reason for this is the difficulty in isolation of Legionella bacteria from clinical samples. In addition, in children clinical symptoms of legionellosis can vary a lot [2]. As this infection can only be transmitted through environmental sources, the main preventing strategy includes proactive environmental surveillance. In addition, this can be combined with antibiotic susceptibility surveillance in high risk areas, such as neonatal and obstetric sink taps in order to prevent the mainly lethal neonatal legionellosis. 
Citation: $\quad$ Alexandropoulou IG, Panopoulou M, Parasidis TA, Konstantinidis T, Constantinidis TC (2015) Preventing Neonatal Legionellosis-The Environmental Surveillance Approach. Clin Microbiol 4: e125. doi:10.4172/2327-5073.1000e125

Page 2 of 2

\section{References}

1. Greenberg D, Chiou CC, Famigilleti R, Lee TC, Yu VL (2006) Problem pathogens: paediatric legionellosis--implications for improved diagnosis. Lancet Infect Dis 6: 529-535.

2. Levy I, Rubin LG (1998) Legionella pneumonia in neonates: a literature review. J Perinatol 18: 287-290.

3. CDC (2003) Guidelines for Environmental Infection Control in HealthCare Facilities (Recommendations of CDC and the Healthcare Infection Control Practices Advisory Committee-HICPAC).

4. EuropeanWorking Group for Legionella Infections (2005) European guidelines for control and prevention of travel-associated Legionnaires' disease.

5. Mavridou A, Smeti E, Mandilara G, Pappa O, Plakadonaki S, et al. (2008) Prevalence study of Legionella spp. contamination in Greek hospitals. Int Environ Health Res 18: 295-304.

6. Velonakis E, Karanika M, Mouchtouri V, Thanasias E, Katsiaflaka A, et al (2012) Decreasing trend of Legionella isolation in a long-term microbial monitoring program in Greek hospitals. Int J Environ Health Res 22: 197-209.

7. Fragou K, Kokkinos P, Gogos C, Alamanos Y, Vantarakis A (2012) Prevalence of Legionella spp. in water systems of hospitals and hotels in South Western Greece. Int J Environ Health Res 22: 340-354.

8. Fragou K, Kokkinos P, Gogos C, Alamanos Y, Vantarakis A (2012) Prevalence of Legionella spp. in water systems of hospitals and hotels in South Western Greece. Int J Environ Health Res 22: 340-354.

9. Alexandropoulou IG, Ntougias S, Konstantinidis TG, Parasidis TA Panopoulou M, et al. (2015) Environmental surveillance and molecular epidemiology of waterborne pathogen Legionella pneumophila in healthcare facilities of Northeastern Greece: a 4-year survey. Environ Sci Pollut Res Int 22: 7628-7640.

10. Shachor-Meyouhas Y, Kassis I, Bamberger E, Nativ T, Sprecher H, et al. (2010) Fatal hospital-acquired Legionella pneumonia in a neonate. Pediatr Infect Dis J 29: 280-281.

11. Wei SH, Chou P, Tseng LR, Lin HC, Wang JH, et al. (2014) Nosocomial neonatal legionellosis associated with water in infant formula, Taiwan. Emerg Infect Dis 20: 1921-1924.

12. Unit for Surveillance and Control of Communicable Diseases (2009) Legionnaires disease in a neonatal unit of a private hospital, Cyprus, December 2008: preliminary outbreak report. Euro Surveill 14
13. Roig J, Rello J (2003) Legionnaires' disease: a rational approach to therapy. J Antimicrob Chemother 51: 1119-1129.

14. von Baum H, Ewig S, Marre R, Suttorp N, Gonschior S, et al. (2008) Community-acquired Legionella pneumonia: new insights from the German competence network for community acquired pneumonia. Clin Infect Dis 46: 1356-1364.

15. Garcia-Vidal C, Carratalà J (2006) Current clinical management of Legionnaires' disease. Expert Rev Anti Infect Ther 4: 995-1004.

16. Pedro-Botet ML, Yu VL (2009) Treatment strategies for Legionella infection. Expert Opin Pharmacother 10: 1109-1121.

17. Almahmoud I, Kay E, Schneider D, Maurin M (2009) Mutational paths towards increased fluoroquinolone resistance in Legionella pneumophila. J Antimicrob Chemother 64: 284-293.

18. Onody C, Matsiota-Bernard P, Nauciel C (1997) Lack of resistance to erythromycin, rifampicin and ciprofloxacin in 98 clinical isolates of Legionella pneumophila. J Antimicrob Chemother 39: 815-816.

19. Bruin JP, Ijzerman EP, den Boer JW, Mouton JW, Diederen BM (2012) Wild-type MIC distribution and epidemiological cut-off values in clinical Legionella pneumophila serogroup 1 isolates. Diagn Microbiol Infect Dis 72: 103-108.

20. Salord JM, Matsiota-Bernard P, Staïkowsky F, Kirstetter M, Frottier J, et al. (1993) Unsuccessful treatment of Legionella pneumophila infection with a fluoroquinolone. Clin Infect Dis 17: 518-519.

21. Kurz RW, Graninger W, Egger TP, Pichler H, Tragl KH (1988) Failure of treatment of legionella pneumonia with ciprofloxacin. J Antimicrob Chemother 22: 389-391.

22. Tsakris A, Alexiou-Daniel S, Souliou E, Antoniadis A (1999) In-vitro activity of antibiotics against Legionella pneumophila isolates from water systems. J Antimicrob Chemother 44: 693-695.

23. Alexandropoulou IG, Parasidis TA, Konstantinidis TG, Constantinidis TC, Panopoulou M (2013) Antibiotic Susceptibility Surveillance of Environmental Legionella Strains: Application of the E-Test to Bacteria Isolated From Hospitals in Greece. J Infect Dis Ther 1:e103.

24. Sandalakis V, Chochlakis D, Goniotakis I, Tselentis Y, Psaroulaki A (2014) Minimum inhibitory concentration distribution in environmental Legionella spp. isolates. J Water Health 12: 678-685. 